\title{
Duale Rezeptorsignale: Wie setzen Ad- häsions-GPCR Signale in Funktion um?
}

VICTORIA ELISABETH GROSS, SIMONE PRÖMEL

INSTITUT FÜR ZELLBIOLOGIE, UNIVERSITÄT DÜSSELDORF

Adhesion GPCR are exceptional receptors due to their functional and structural diversity. A key to their function/signalling, setting them apart from other GPCR, is their extraordinarily large, complex $\mathrm{N}$ terminus, via which they mediate different molecular mechanisms and integrate diverse biological functions. Here, we discuss dual modes of adhesion GPCR action and how they translate into physiological functions: activation of $G$ protein pathways and signals solely elicited by the $\mathrm{N}$ terminus.

DOI: $10.1007 / \mathrm{s} 12268-021-1625-1$

(C) Die Autorinnen 2021

- Um miteinander und mit der Umwelt kommunizieren zu können, nutzen unsere Zellen eine Vielzahl an Oberflächenmolekülen. Eine Familie dieser Moleküle sind G-Protein-gekoppelte Rezeptoren (GPCR), welche verschiedenste Stimuli - wie beispielsweise Hormone, Metabolite, oder auch Photonen - außerhalb der Zelle detektieren und in Form von Signalen ins Zellinnere

übersetzen. Aufgrund ihrer Lage auf der Zelloberfläche und ihrer damit verbundenen guten Zugänglichkeit sowie ihrer Beteiligung an einer Vielzahl von (patho)physiologischen Prozessen werden GPCR oft als pharmakologische Zielstrukturen genutzt. Jedoch ist das pharmakologische Potenzial nicht von allen GPCR gleichermaßen erschlossen. Zu den kaum erschlossenen Rezeptoren gehören die

A LPHN3

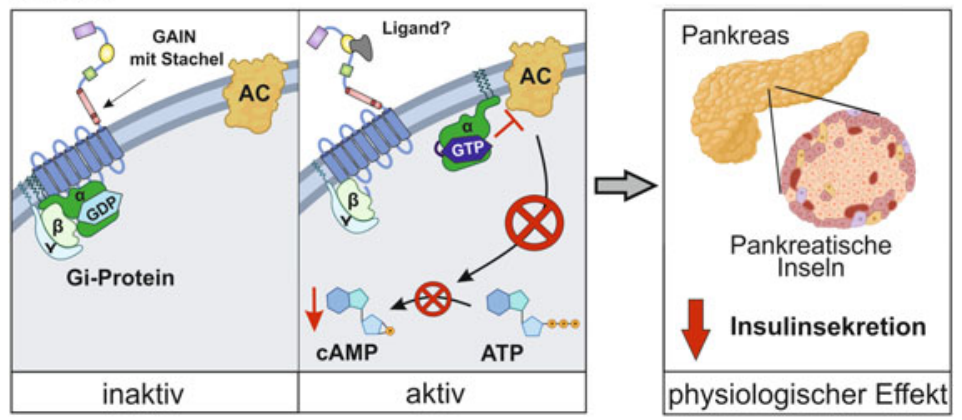

B LAT-1

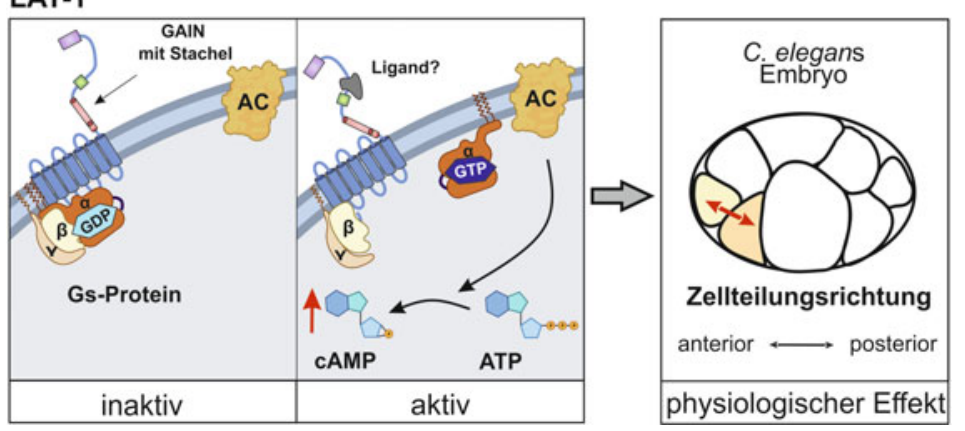

Adhäsions-GPCR, welche mit 33 Paralogen im Menschen die zweitgrößte Klasse der GPCR bilden. Ihre diversen Funktionen in neurobiologischen, immunologischen, entwicklungsbiologischen oder metabolischen Prozessen [1] zeigen ihre Bedeutung für den Organismus. Zudem deuten Defekte in bestimmten Vertretern, die zu schwerwiegenden Erkrankungen wie dem Usher-Syndrom, der bilateralen frontoparietalen Polymikrogyrie oder der vibratorischen Urtikaria führen [2], ihre klinische Relevanz an.

\section{Adhäsions-GPCR vermitteln verschiedenste Funktionen}

Adhäsions-GPCR sind besondere GPCR. So sind sie nicht nur insgesamt in ihren Funktionen divers, auch ein einzelner Rezeptor spielt meist mehrere unterschiedliche Rollen in verschiedenen Kontexten. Um diese zu realisieren, besitzen die Moleküle neben den typischen strukturellen GPCR-Charakteristika, wie der Siebentransmembrandomäne und einem intrazellulären C-Terminus, außergewöhnlich große und komplexe extrazelluläre N-Termini (Abb. 1). Sie enthalten je nach Adhäsions-GPCR eine spezifische Kombination an Domänen, wie beispielsweise Cadherin-, Laminin-, EGF-(epidermal growth factor), Ig-(Immunglobulin), oder PentraxinDomänen. Aufgrund der adhäsiven Eigenschaften dieser Domänen war schon früh bekannt, dass Adhäsions-GPCR nicht nur Signale in Zellen weiterleiten, sondern auch

4 Abb. 1: Cis-Signale von Latrophilinen und ihre physiologische Funktionen. A, Der von LPHN3 vermittelte $\mathrm{G}_{i}$-Signalweg (links) führt nach Rezeptoraktivierung durch die Stachelsequenz über Inhibition der Adenylylcyclase (AC) zu einem verringerten cAMP-Spiegel in Zellen (Mitte) und zu einer Reduktion der Insulinausschüttung aus pankreatischen Inseln (rechts). B, Das Latrophilin-Homolog LAT-1 in Caenorhabditis elegans aktiviert einen $\mathrm{G}_{\mathrm{s}}$-Signalweg (links), wodurch die Produktion von cAMP erhöht wird (Mitte), was die korrekte Ausrichtung der anterior-posterioren (a-p) Zellteilungsebene im Embryo bewirkt (rechts). Erstellt mit BioRender. 
Adhäsion vermitteln. Eine Domäne ist fast allen Adhäsions-GPCR gemeinsam: die GAIN(GPCR-autoproteolysis-inducing)-Domäne, in der sich in vielen Fällen die GPCR proteolysis site (GPS) befindet, die der Ort einer autokatalytischen Spaltung ist [3]. Obwohl etliche Rezeptoren an dieser Stelle prozessiert werden, ist ihre Bedeutung noch nicht geklärt.

Die vielen unterschiedlichen Domänen in den N-Termini von Adhäsions-GPCR deuten auf ein großes Repertoire an Liganden hin. Jedoch sind bislang nur einige identifiziert, bei denen es sich meist um Komponenten der extrazellulären Matrix oder um Moleküle auf benachbarten Zellen handelt [4]. Die Aktivierung von Adhäsions-GPCR ist derzeit noch nicht hinreichend verstanden. Es ist allerdings bekannt, dass sie mechanosensitive Eigenschaften besitzen und dass sie über einen gebundenen Agonisten aktiviert werden, der in ihren N-Termini gelegen ist und Stachelsequenz genannt wird (Abb. 1, [5]). Die Rezeptoraktivierung führt dabei, wie bei anderen GPCR auch, meist zu einem klassischen Signal, das intrazellulär über heterotrimere G-Proteine vermittelt wird.

All diese strukturellen und funktionellen Eigenschaften von Adhäsions-GPCR deuten - zusätzlich zu den klassischen Mechanismen - auf diverse ungewöhnliche molekulare Funktionen und Signale hin. Wie Adhäsions-GPCR ihre verschiedenen Funktionen auf molekularer Ebene realisieren und vor allem, wie die großen, komplexen N-Termini als Drehscheibe der Rezeptoren die Funktionen koordinieren, ist derzeit Gegenstand aktiver Forschung.

\section{Signale in die Zelle - cis signalling}

Viele Adhäsions-GPCR sind hochkonserviert, es existieren Vertreter in Vertebraten sowie Invertebraten. Diese lassen sich gut als „prototypische“ Adhäsions-GPCR nutzen, um ein detailliertes Verständnis darüber zu erlangen, welche Signale die Rezeptoren vermitteln und wie sie diese in physiologische Funktionen übersetzen. Eine Gruppe solcher Adhäsions-GPCR sind beispielsweise die Latrophiline. Während in Säugern drei Homologe existieren (Lphn1-3/Adgrl1-3), weist der Fadenwurm Caenorhabditis elegans zwei Vertreter auf (lat-1, lat-2). Alle drei Latrophiline in Säugern besitzen, wie viele andere Adhäsions-GPCR auch, verschiedene Funktionen. So spielen LPHN1 und LPHN3 sowohl in Neuronen eine wesentliche Rolle $[6,7]$, als auch in pankreatischen Inseln. In
Letzteren konnte unsere Gruppe mit zeigen, dass LPHN3 die Insulinsekretion moduliert - die Aktivität des Rezeptors reduziert die Ausschüttung des Hormons [8]. Dabei ist zwar noch nicht bekannt, welche Moleküle in diesem Fall an den Rezeptor binden, jedoch wird er über die im N-Terminus gelegene Stachelsequenz aktiviert. Der so aktivierte Rezeptor vermittelt über eine $\mathrm{G}_{\mathrm{i}}$-Protein-Kaskade ein Signal in die Zelle (cis-Signal). Das aktivierte $\mathrm{G}_{\mathrm{i}}$-Protein (GTP-bindend) bewirkt eine Inhibition der Adenylylcyclase, welche im aktiven Zustand ATP zum second messenger zyklisches AMP (CAMP) umwandelt. Die Inhibition führt somit zu einer Reduktion des CAMP, was wiederum über mehrere, noch unbekannte Schritte, zu einem Herabsenken der Insulinausschüttung führt (Abb. 1A).

Interessanterweise unterscheidet sich dieses LPHN3-Molekül in pankreatischen Zellen in seinem Aufbau von dem in Neuronen. Verschiedene Splicevarianten führen hier zu zwei Rezeptorversionen, welche sich hauptsächlich im Bereich der Siebentransmem- brandomäne und dem intrazellulären C-Terminus unterscheiden. Diese Unterschiede bewirken, dass die Variante in pankreatischen Zellen $\mathrm{G}_{\mathrm{i}}$-Signale vermittelt, die in Neuronen jedoch eher $G_{q / 11}$-Signale. Dies ist ein Beispiel dafür, wie Adhäsions-GPCR die vielfältigen Funktionen auf molekularer Ebene realisieren.

Erste in vitro-Untersuchungen geben Hinweise darauf, dass auch LPHN1 eine Rolle in der Modulation der Insulinausschüttung in pankreatischen Inseln spielt, der Rezeptor jedoch potenziell als eine Art Gegenspieler des LPHN3 über einen $\mathrm{G}_{\mathrm{s}}$-Protein-Signalweg eine Erhöhung der intrazellulären CAMPSpiegel bewirkt [8].

Die klassische Signaltransduktion eines Adhäsions-GPCR in eine Zelle über einen G-Protein-Signalweg ist hochkonserviert und ebenfalls Latrophiline in C. elegans besitzen sie. So wird das C. elegans-Latrophilin-Homo$\log$ LAT-1, wie die Säugerhomologe auch, durch eine intrinsisch in der GAIN-Domäne gelegene Stachelsequenz aktiviert, was zeigt, wie konserviert auch dieser Mechanismus ist

\section{Hier steht eine Anzeige.}

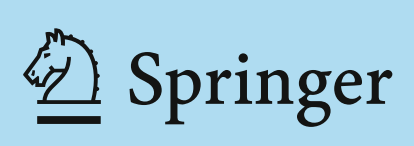




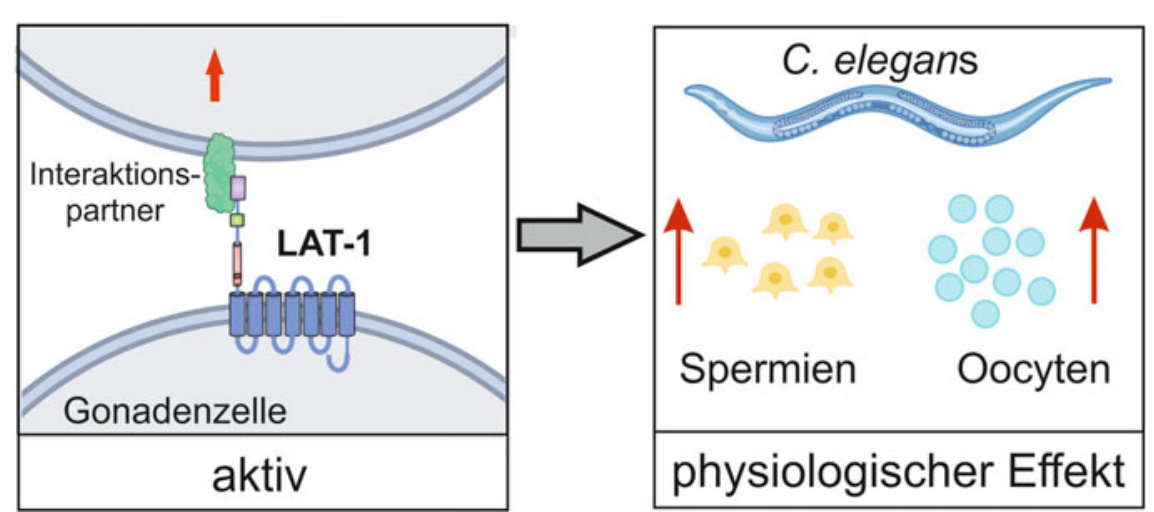

$\Delta$ Abb. 2: Latrophilin steuert über ein trans-Signal (links) die Keimzellzahl in Caenorhabditis elegans (rechts). Das trans-Signal wird über einen bislang unbekannten Mechanismus in die Nachbarzelle geleitet. Erstellt mit BioRender.

[9]. Die Aktivierung von LAT-1 führt über eine $\mathrm{G}_{\mathrm{s}}$-Protein-Signalkaskade zur Aktivierung der Adenylylcyclase (durch das GTPbindende $\mathrm{G}_{\mathrm{s}}$-Protein), welche eine Erhöhung der intrazellulären Konzentration des second messengers cAMP bewirkt. Über diesen Mechanismus steuert LAT-1 im frühen Embryo bestimmte Aspekte der Zellpolarität (Abb. 1B, [9-11]). Nematoden mit einer lat-1-Defizienz zeigen schwerwiegende Defekte in der Anordnung der Teilungsspindeln einiger sich symmetrisch teilender Zellen in anterior-posteriorer Richtung. Dies führt zu falschen Zell-Zell-Kontakten und zum Tod eines großen Teils der betroffenen Individuen [10]. Die Signale von LAT-1 sorgen dafür, dass die Zellen die korrekte Teilung in anterior-posteriorer Richtung erhalten.

\section{Signale in andere Zellen - trans signalling}

Neben den klassischen G-Protein-vermittelten Signalen von Adhäsions-GPCR in eine Zelle, die auch als cis-Funktion bezeichnet werden, gewinnt zunehmend eine zweite Form der Aktivität eine Bedeutung, die transFunktion. Diese ist sehr ungewöhnlich für GPCR und wir und andere Arbeitsgruppen konnten zeigen, dass sie keine G-ProteinSignale in die Zelle beinhaltet, sondern ausschließlich über den großen N-Terminus der Rezeptoren vermittelt wird. So hat der N-Terminus des Latrophilin-Homologs LAT-1 in C. elegans eine Funktion in der Bildung beziehungsweise der Funktion von Keimzellen [11]. Hierbei ist es ausreichend, wenn der $\mathrm{N}$-Terminus allein an der Membran verankert ist. Der N-Terminus, welcher auf den Zellen der Gonaden vorkommt, hat dann einen Effekt auf die benachbart liegenden (Keim-)Zellen (in trans) und bewirkt, dass die korrekte Anzahl an voll funktionsfähigen Keimzellen gebildet wird (Abb. 2). Bei C. elegans handelt es sich um Hermaphrodi- ten, sie produzieren also Oocyten und Spermien. Beide sind von der Kontrolle durch den N-Terminus von LAT-1 betroffen.

Auch für andere Adhäsions-GPCR, wie beispielsweise GPR126, wurde eine trans-Funktion beschrieben [12], was auf einen generellen Mechanismus hindeutet.

\section{Fazit}

Die Eigenschaft, duale Funktionen - cis und trans - zu vermitteln, ist außergewöhnlich für GPCR. Sie scheint jedoch eine Gemeinsamkeit etlicher Adhäsions-GPCR zu sein und zeigt, wie diese Rezeptoren verschiedene physiologische Funktionen über ihre großen und komplexen N-Termini realisieren können. Zukünftige Studien werden zeigen, wie die einzelnen Adhäsions-GPCR cis- und transFunktion im Detail integrieren und kontextspezifisch umsetzen.

\section{Literatur}

[1] Hamann J, Aust G, Arac D et al. (2015) International union of basic and clinical pharmacology. XCIV. Adhesion $G$ protein-coupled receptors. Pharmacol Rev 67: 338-367 [2] Schöneberg T, Liebscher I (2021) Mutations in G protein-coupled receptors: mechanisms, pathophysiology and potential therapeutic approaches. Pharmacol Rev 73: 89-119
[3] Prömel S, Langenhan T, Arac D (2013) Matching structure with function: the GAIN domain of adhesion-GPCR and PKD1-like proteins. Trends Pharmacol Sci 34: 470-478 [4] Bondarev AD, Attwood MM, Jonsson J et al. (2020) Opportunities and challenges for drug discovery in modulating Adhesion G protein-coupled receptor (GPCR) functions. Expert Opin Drug Discov 15: 1291-1307

[5] Liebscher I, Schön J, Petersen SC et al. (2014) A tethered agonist within the ectodomain activates the adhesion $\mathrm{G}$ protein-coupled receptors GPR126 and GPR133. Cell Rep 9: 2018-2026

[6] Sando R, Jiang X, Sudhof TC (2019) Latrophilin GPCRs direct synapse specificity by coincident binding of FLRTs and teneurins. Science 363: eaav7969

[7] Silva JP, Lelianova VG, Ermolyuk YS et al. (2011) Latrophilin 1 and its endogenous ligand Lasso/teneurin-2 form a high-affinity transsynaptic receptor pair with signaling capabilities. Proc Natl Acad Sci U S A 108: 12113-12118 [8] Röthe J, Thor D, Winkler J et al. (2019) Involvement of the adhesion GPCRs latrophilins in the regulation of insulin release. Cell Rep 26: 1573-1584

[9] Müller A, Winkler J, Fiedler F et al. (2015) Oriented cell division in the $C$. elegans embryo is coordinated by G-protein signaling dependent on the adhesion GPCR LAT-1. PLoS Genet 11: e1005624

[10] Langenhan T, Prömel S, Mestek L et al. (2009) Latrophilin signaling links anterior-posterior tissue polarity and oriented cell divisions in the C. elegans embryo. Dev Cell 17: 494-504

[11] Prömel S, Frickenhaus M, Hughes S et al. (2012) The GPS motif is a molecular switch for bimodal activities of adhesion class G protein-coupled receptors. Cell Rep 2: 321331

[12] Patra C, van Amerongen MJ, Ghosh S et al. (2013) Organ-specific function of adhesion protein-coupled receptor GPR126 is domain-dependent. Proc Natl Acad Sci U S A 110: 16898-16903

Funding note: Open Access funding enabled and organized by Projekt DEAL. Open Access: Dieser Artikel wird unter der Creative Commons Namensnennung 4.0 International Lizenz veröffentlicht, welche die Nutzung, Vervielfältigung, Bearbeitung, Verbreitung und Wiedergabe in jeglichem Medium und For
erlaubt, sofern Sie den/die ursprünglichen Autor(en) und die Quelle ordnungsgemäß nennen, einen Link zur Creative Commons Lizenz beifügen und angeben, ob Änderungen vorgenommen wurden. Die in diesem Artikel enthaltenen Bilder und sonstiges Drittmaterial unterliegen ebenfalls der genannten Creative Commons Lizenz, sofern sich aus der Abbildungslegend nichts anderes ergibt. Sofern das betreffende Material nicht unter der genannten Creative Commons Lizenz steht und die betreffende Handlung nich nach gesetzlichen Vorschriften erlaubt ist, ist für die oben aufgeführt Rechteinhabers einzuholen. Weitere Details zur Lizenz entnehmen Sie bitte der Rechteinhabers einzuholen. Weitere Details zur Lizenz entnehmen Sie bitte de
Lizenzinformation auf http://creativecommons.org/licenses/by/4.0/deed.de.

Korrespondenzadresse:

Prof. Dr. Simone Prömel

Institut für Zellbiologie

Heinrich-Heine-Universität

Universitätsstraße 1

D-40225 Düsseldorf

proemel@uni-duesseldorf.de

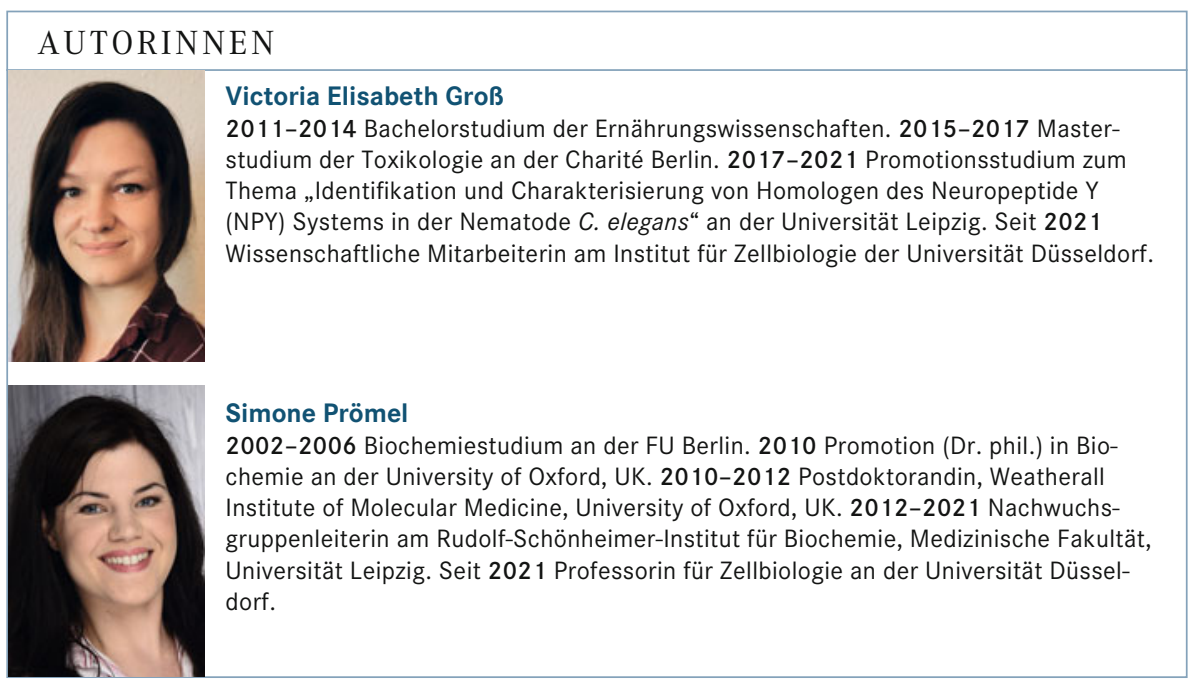

